\title{
Design and Simulation of Paper Roll Packaging Workstation Based on RobotStudio
}

\author{
Biqiong Li and Chaoan Liu \\ College of Automobile and Mechanic Engineering, Changsha University of Science and Technology, Changsha, China
}

\begin{abstract}
Taking ABB robot as the research object, the simulation workstation of paper roll packaging was established by SolidWorks three-dimensional modeling and Robot Studio simulation software. According to the technological process of paper roll packaging, the overall spatial layout of the robot paper roll packaging workstation was constructed. SolidWorks was used to model the packaging machine and other equipment, I/O signals and Smart components were created, and the robot packaging simulation program was compiled offline. The simulation results show that the paper roll packaging workstation can realize the robot packaging process.
\end{abstract}

Keywords—-three-dimensional modeling; paper packaging; Robot Studio; virtual simulation

\section{INTRODUCTION}

Since the birth of the first robot in the 1960s, industrial robotics technology has developed rapidly ${ }^{[1-2]}$. Robots of various forms and functions have emerged one after another, freeing the operators from heavy, monotonous and dangerous work places. At present, although most of the paper roll packaging machines used in domestic papermaking industry have realized automation, they still need to be manually placed inside and outside the end face ${ }^{[3]}$. In order to solve the above problems, this paper designs a paper roll packaging system based on ABB robot. Two robots grab the head on the assembly line and place it on the head placement machine to package the paper roll. The third robot pastes the label on the paper roll to complete the paper roll packaging ${ }^{[4]}$.

\section{Design Of Workstation Simulation System}

\section{A. Building Simulation Platform}

Robot Studio-based simulation system for robotic packaging workstation is shown in Figure 1. In Robot Studio, the three-dimensional models of IRB6700, IRB4600 and SolidWorks are imported ${ }^{[5]}$, and the spatial layout of equipment is adjusted. Tool data, workpiece coordinates, I/O signals and Smart components are set up. Through debugging program, the robot can automatically complete the whole process of paper roll head placement and labeling.

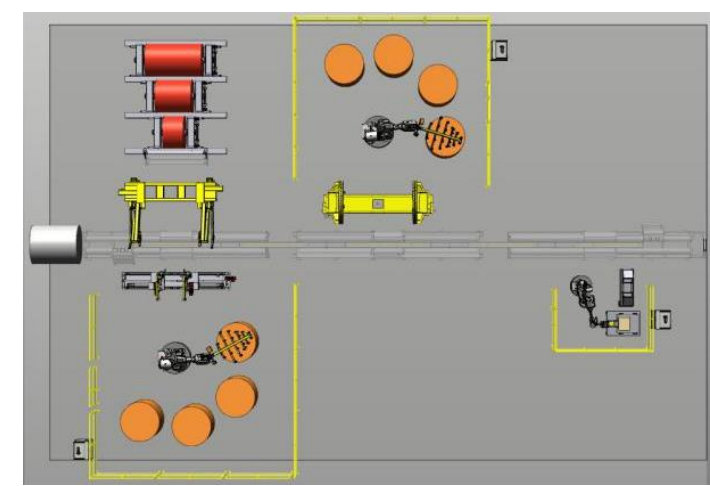

FIGURE I. SIMULATION WORKSTATION OF PAPER ROLL PACKAGING

\section{B. Design of Robot Terminal Claw}

The workstation robot mainly completes the grasping and pasting of internal and external heads and trademarks. Considering the light and thin grasping objects, it adopts the principle of vacuum pump adsorption to achieve. The suction cup of the end of the head robot adopts a double-sided suction method, which can suck two heads at the same time. After the end sucker is modeled by SolidWorks, it is imported into Robot Studio to become a new mechanical device and installed on the six axes of the robot. Because the robot grasps different objects, two kinds of end sucker grippers are designed, as shown in Figure 2.

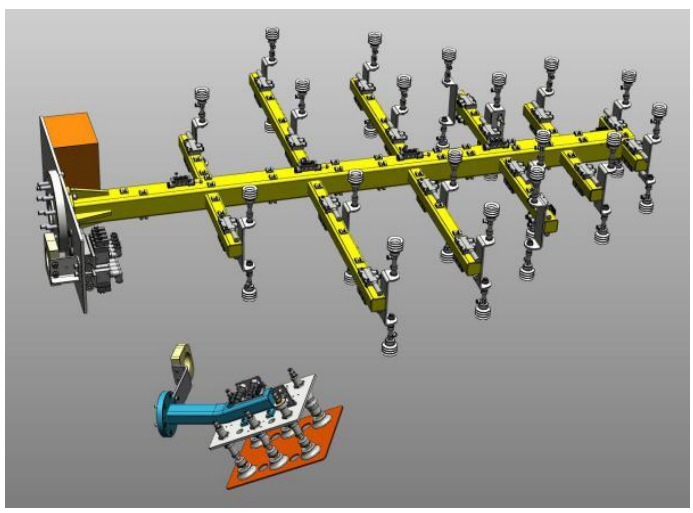

FIGURE II. ROBOT END SUCKER 


\section{ESTABLISHMENT OF TOOL AND WORKPIECE COORDINATES}

\section{A. Tool Coordinates}

Tool coordinates are the position Parameter of the tool center point (TCP) at the end of the robot. The motion of the robot is the coincidence of the TCP point and the path point of the robot. In $\mathrm{t}$ his paper, the robot needs to establish multiple tool coordinates and c hoose different tool coordinates in motion, which can achieve different motion effects. Specific settings are shown in Figure 3.

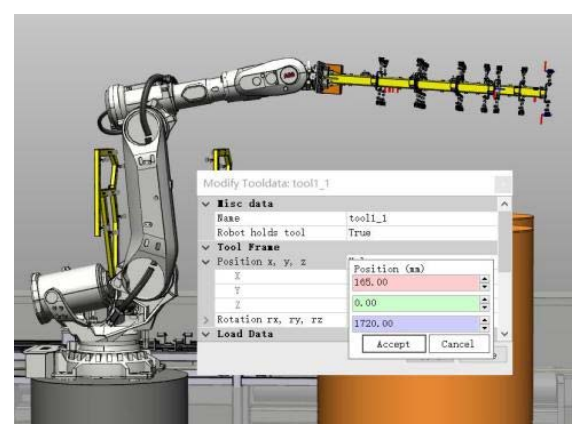

FIGURE III. ESTABLISHMENT OF TOOL COORDINATES

In the name of creating tool data, enter the name you want to establish. Select the tool coordinate frame to be related to the geodetic coordinate system. In the tool coordinate frame, set its tool coordinate system.

\section{B. Work Piece Coordinates}

Work piece coordinate system is the coordinate system used in programming, including user coordinate frame and work piece coordinate frame. Because the label and head stack will be consumed continuously in this paper, the work piece coordinate system is set to coincide with the robot coordinate system. It is convenient to read the relative position of the label and head stack with the robot. The work piece coordinate of the inner head robot is set as shown in Fig. 4, and the principle of the work piece coordinate setting of the other two robots is the same.

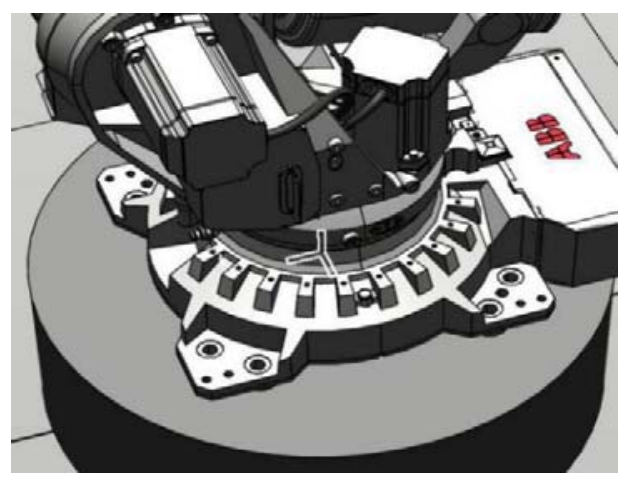

FIGURE IV. ESTABLISHMENT OF WORK PIECE COORDINATES

\section{ESTABLISHMENT OF I/O Signal AND SMART COMPONENT}

$\mathrm{I} / \mathrm{O}$ signal is used to realize the communication between robot and external equipment, Smart component is an efficient tool to achieve animation effect in Robot Studio. Common signals provided by standard I/O boards are digital input, analog input, digital output, analog output and conveyor chain tracking ${ }^{[6]}$. $\mathrm{I} / \mathrm{O}$ board is a device located on the field bus. Its function is to correlate the $\mathrm{I} / \mathrm{O}$ signal of Smart component with the I/O signal of the robot, that is, the input signal of the robot end is the output signal of the Smart component, and the output signal of the robot end is the input signal of the Smart component.

This workstation uses four I/O boards named PANEL, DRV 1, D652 and PLC. The I/O address range is 0-47. It is connected to Fieldbus Device Net and Pr ofibus DP, a s shown in Figure 5.

\begin{tabular}{|c|c|c|c|c|}
\hline \multicolumn{5}{|l|}{ Configuration - V/O System $x$} \\
\hline Type & Nase & Type of Signal & Assigned to Device & Signal \\
\hline Access Level & AS1 & Disital Input & PANELL & Automatic \\
\hline Cross Connection & AS2 & Disital Input & PANEL & Automatic \\
\hline Device Trust Level & AUTO1 & Digital Input & PANEL & Automatic \\
\hline DeviceNet Command & AUT02 & Digital Input & PANEL & Autoeatic \\
\hline DeviceNet Device & CHI & Digital Input & PANEL & Run Chair \\
\hline & $\mathrm{CH}_{2}$ & Digital Input & PANEL & Run Chair \\
\hline DeviceNet Internal Device & DRVIBRAKE & Digital Output & DRV_1 & Brake-rel \\
\hline Bther:let/IP Comand & DRV1BRAKEFB & Digital Input & DRY_ 1 & Brake Fec \\
\hline EtherNet/IP Device & DRVIBRAKEOK & Digital Input & $\mathrm{DRV}_{-} 1$ & Brake Vol \\
\hline Industrial Netrork & DRV1CHAIN1 & Digital Output & DRV_I & Chain 11 \\
\hline PROFINBT Common Data & DRV1CHAIN2 & Digital Output & DRV_ 1 & Chain 2 I \\
\hline PROFINET Internal Device & DRVIEXTCONT & Digital Input & DRV_ 1 & External \\
\hline Route & DRVIFASI & Digital Input & DRV 1 & Drive Uni \\
\hline Signal & DRV1FAN2 & Digital Input & $\mathrm{DRV}_{-} 1$ & Drive Uni \\
\hline & DRVIK1 & Digital Input & $\mathrm{DRV}_{-} \mathrm{I}$ & Contactor \\
\hline Signal Safe Level & DRV1K2 & Digital Input & DRV_1 & Contactor \\
\hline \multirow{8}{*}{ System Output } & DRVILTWI & Digital Input & $\mathrm{DRV}_{-1}$ & Linit Svi \\
\hline & DRVILIV2 & Digital Input & DRV_ 1 & Linit Svi \\
\hline & DRVIPANCH1 & Digital Input & DRV_ 1 & Drive Vol \\
\hline & DRV1PANCH2 & Digital Input & $\mathrm{DRV}_{-} 1$ & Drive Vol \\
\hline & DRVIPTCEXI & Digital Input & DRV_ 1 & External \\
\hline & DRVIPTCINT & Dirital Input & DRV_ 1 & Yotor tes \\
\hline & DFV1SPEED & Digital Input & DRV_ 1 & Speed Sig \\
\hline & DRVITEST1 & Digital Input & $\mathrm{DRV}_{-} 1$ & Run chair \\
\hline
\end{tabular}

FIGURE V. I/O SIGNAL CONFIGURATION OF ROBOT

This workstation needs to build three S mart components to realize the simulation of the whole workstation, including the cartoon effect of the end sucker grabbing the head or trademark, the transport device that drives the paper roll moving, the head placer and the hot press.

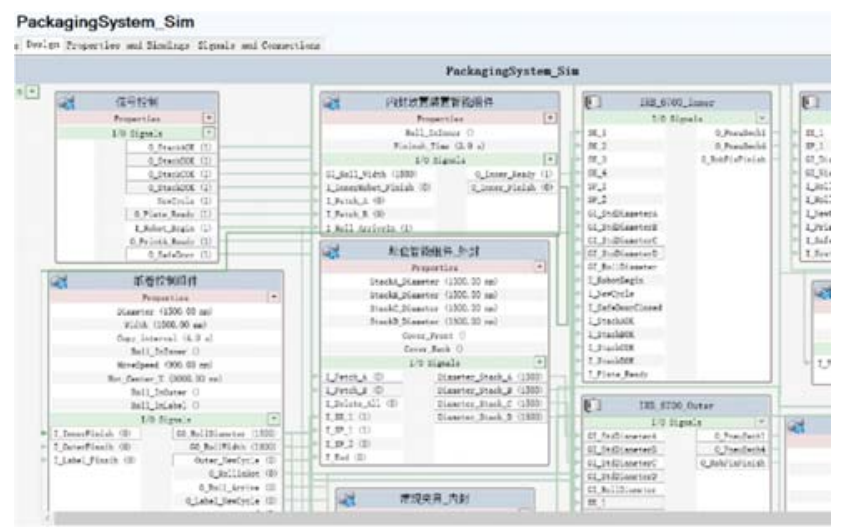

FIGURE VI. ROBOT SIGNAL CONNECTION DIAGRAM 
program. The wo rk effect of the w orkstation is shown in

LineSensor, Attacher, Detacher and LogicGate [NOT] sub-components are adde d to the end sucker, PlanSensor, PoseMover and LogicSRLatch sub-components are added to the tra nsport device, LineSensor, Attacher, Sink and PoseMover sub-components are added to the head. placer and the hot press, and then the properties and signals of each sub-component are set. The robot signal is connected to the Smart component signal, as shown in Figure 6.

\section{System PROGRAMMING AND SimUlation}

\section{A. Programming}

Before compiling the robot program, it is necessary to determine the target point of the robot motion. When compiling the program, each target point is co nnected in series in different motion modes through the robot motion control instructions to achieve the desired motion path. Rapid language program is composed of modules. FetchHalf (capturing and installing headers) and Functions are established in the $r$ obot program of this workstation.(Function),Interrupts (Interrupt), TeachPrg (Debugging), UpdataPar (Update Parameters), VariableMod (Storage Data) six modules, each module is composed of the corresponding routine.

Some routine procedures for the robot to grab the hea are as follows:

\section{PROC FetchSideAA() \\ CLEAR T;}

lbelA1:

IF T $>3$ THEN

TPWRITE "The stack A runs out or

the vacuum pump stops working.";

T PWRITE "Please Check!";

$$
\text { GoToSa feSpot; }
$$

GoToHomePos

\section{EXIT;}

\section{ENDIF}

ConfJ $\backslash O n$;

A ;

Move $\backslash$ Conc,FBeginSrchPosAA,v2000,z5, gripper

\section{chPosAA,v500, gripperA;}

SearchL \PStop,I_LaserSensorA,TmpVerPoint,FSr

\section{B. Simulation Analysis}

This workstation builds a paper roll packaging production line system based on robots. Without affecting the quality and safety of packaging, the most important factor affecting the workstation rhythm is the speed of TCP movement of robots. Increasing the speed of TCP can improve the production rhythm of workstations.

The speed of TCP is $1000 \mathrm{~mm} / \mathrm{s}$ when the robot grabs the head, $1500 \mathrm{~mm} / \mathrm{s}$ when it grabs the trademark and $2000 \mathrm{~mm} / \mathrm{s}$ when it is idle. After completion, click the "Play" button of the "Simulation" tab. The s ystem executes the paper roll packaging task according to the preset
Figure 7. According to the timer, it takes about 98 seconds to complete a piece of Paper roll packaging. The efficient work of the robot off-line programming workstation provides a powerful guarantee for the production efficiency of the paper roll packaging production line.

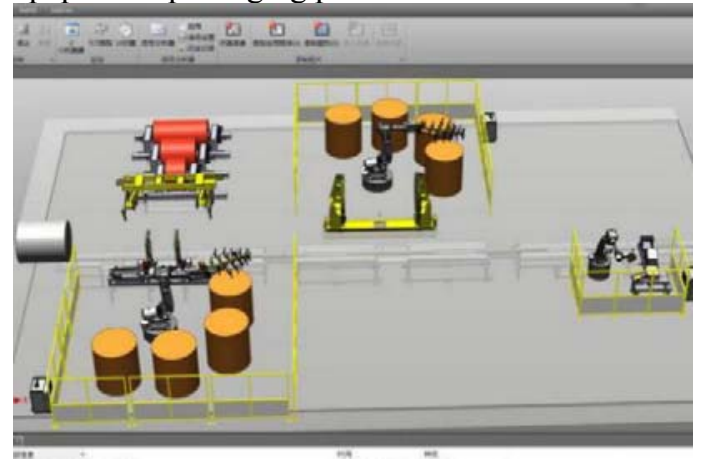

(a)Robot Moves to the Initial Position

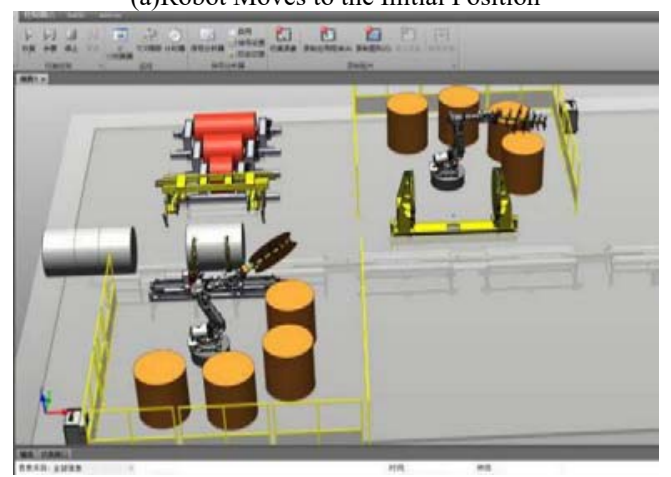

(b) Robot Grabs Inner Head

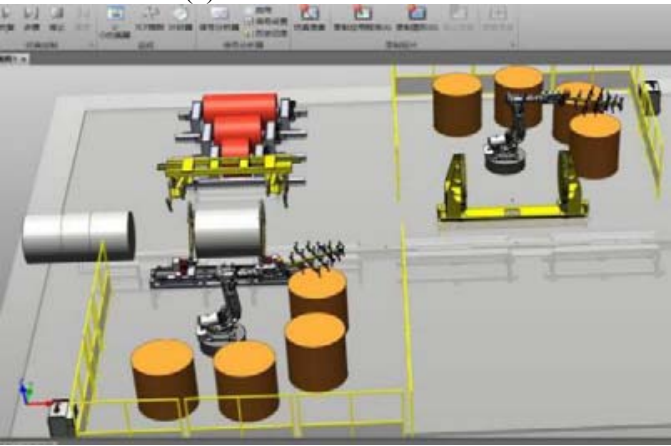

(c) Places Inner Head

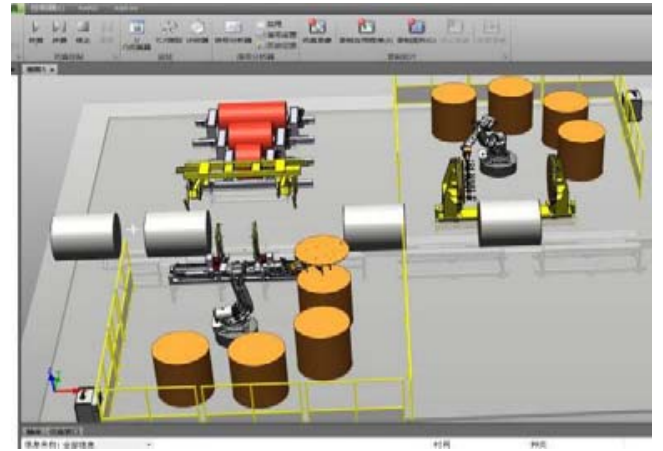

(d) Put the outer head on the hot-press plate 
transmission, which is convenient for field debugging. It provides a feasible basis for the design of robot packaging workstation and helps the robot in the field of paper roll packaging. Replacing manual labor and further improving the degree of automation in papermaking industry have important guiding significance for guiding actual production.

\section{REFERENCES}

[1] Li Kunquan, Shao Fengxiang. Design of automatic packing and palletizing robot control system $[\mathrm{J}]$. Mechanical design and manufacture, 2017 (4): 259-262.

[2] Guo Jinheng, Guo Zonghua, Yin Zhihui, et al. Applic ation of Industrial Robot in Metallurgical Automatic Packing and Palletizing Production Line System [J]. Intelligent Robot, 2018 (04): 42-45.

(e) Installation of external head

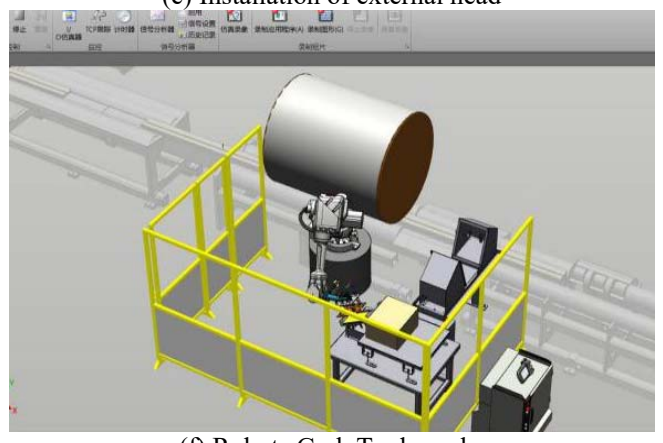

(f) Robots Grab Trademarks

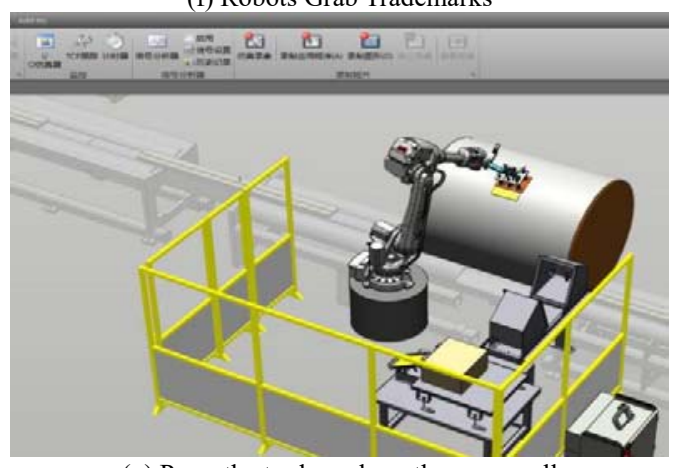

(g) Press the trademark on the paper roll

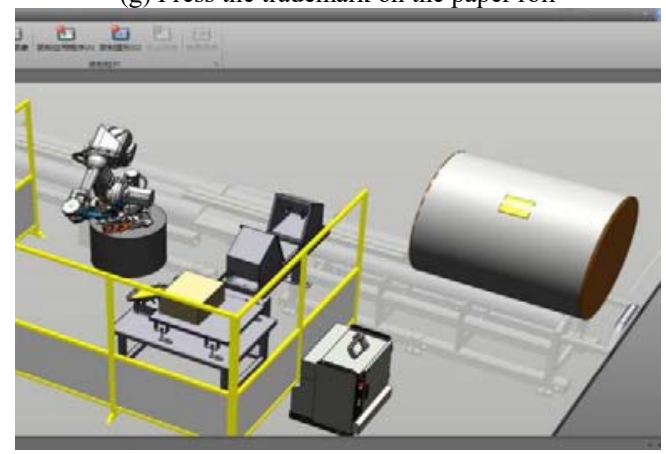

(h) Paper roll for completion of packaging

[3] Hu Chao. Application of Intelligent System in Automatic Paper Roll Packing Machine [J]. China Paper Industry, 2013 (20).

[4] Hu Lianhua, Li Xinping, Tang Wei. Design of automatic packaging system for paper rolls based on industrial robots [J]. China Paper Mill, 34 (4).

[5] Application of secondary development of Wang Rugeng, Fu Gaosheng, Chen Hongling, et al. SolidWorks in off-line programming of industrial robot abrasive belt grinding and polishing [J].Manufacturing Automation,2018,v.40(05): 135-138.

[6] Bai lei,Hou Wei, Zhang Xiaojie. Robot Studio-based Robot Handling Workstation Design and $\mathrm{Si}$ mulation $[\mathrm{J}]$. Foreign Electronic Measurement Technology, 2018 (6).

FIGURE VII. WORKSTATION OPERATION EFFECT

\section{CONCLUSION}

In this paper, Robot Studio is used to build a robot packaging workstation. Through simulation, the programming effect can be seen intuitively. Robot program data can be $t$ ransmitted to the $r$ eal robot through data 